\title{
Fssc22000-1. Una Visión del Sistema de Certificación en InOCUidad de Alimentos
}

\section{Fssc22000-1. A View of Food Safety Certification System}

\author{
Laura Marcela Bernal Saenz \\ Escuela de Ciencias Básicas Tecnología e Ingeniería, Universidad Nacional \\ Abierta y a Distancia - UNAD, Ibagué, Colombia \\ Laura.bernaleunad.edu.co
}

Recibido: 14 /07/2013 • Aprobado: 02/09/2013

\section{RESUMEN}

FSSC22000 fue desarrollado en Europa en el año 2009 y surgió del interés de grandes compañías como Nestlé, Danone, Unilever, Coca Cola, Kraft Products, entre otras, de adoptar un nuevo modelo de certificación en inocuidad para todos sus proveedores de materias primas. Está basado en la integración de la Norma ISO 22000 y la guía de pre-requisitos generales BSI-PAS 220. El estándar ha sido promovido por la Confederación Europea de Industrias de Alimentación y Bebidas (CIAA), y aprobado por Iniciativa Global para la Seguridad Alimentaria (GFSI). Desde el año 2009 hasta el 28 de septiembre de 2015 (fecha de estudio) se habían certificado en el mundo 10.595 plantas de producción, encontrándose Nestlé, Cargill y Mondelez en los tres primeros puestos; Estados Unidos liderando la lista; Japón, China, India y México ocupando los 5 primeros lugares yAmérica Latina aportando el 10,9\% de compañías certificadas, destacándose México con mayor número. El objetivo principal de este artículo es dar a conocer el último sistema de inocuidad desarrollado para las plantas manufactureras de la cadena de alimentos, basado en los criterios que establece la FFSC, Fundación encargada de administrar el modelo en todo el mundo. La metodología utilizada recoge dos elementos fundamentales para su desarrollo: la experiencia de la autora en implementación de sistemas de gestión y la recopilación de fuentes documentales de diferentes autores y organizaciones.

Palabras clave: BSI-PAS220, FSSC22000, FSSC22000-1, sistema de inocuidad alimentaria.

\section{AbstRact}

FSSC22000 was developed in Europe in 2009 and grew out of the interest of large organizations such as Nestlé, Danone, Unilever, Coca Cola, Kraft Products, among others, to adopt a new model safety certification for all its suppliers of raw materials. It is based on the integration of ISO 22000 and the guidance of general prerequisites BSI-PAS 220. The standard has been promoted by the European Confederation of Food and Drink Industries (CIAA), and approved by the Global Food Safety Initiative (GFSI).Since 2009 until September 28th 2015, 10595organizations in the food chain worldwide has been 
certificated. Nestle, Cargill y Mondelez which occupy the first three places. Globally, the United States leads the list, Japan, China, India, and Mexico occupy the top 5. Latin America contributes only 10,9\%, being Mexico the country with the most organizations certificated.The main objective of this article is to present the latest safety food system developed for organizations in the food chain, based on criteria established by the FFSC; foundation responsible for manage the model worldwide. The methodology includes two basic elements for development; the experience on implementing management systems and compilations author of documentary sources by different authors and organizations.

Key words: BSI-PAS220, FSSC22000, FSSC22000-1, food safety certification.

\section{INTRODUCCIÓN}

El mundo ha avanzado y llegar al nivel en el que las organizaciones, en particular las manufactureras de alimentos, se encuentran actualmente, ha implicado una serie de cambios y evolución de pensamiento acerca de lo que se persigue al llegar al consumidor y tratar de conquistarlo [1]. Años atrás, no existía ningún interés en cuanto a si las organizaciones eran responsables con el medio ambiente, si respetaban las condiciones de trabajo de los colaboradores o más aún si las condiciones de procesamiento eran suficientes para certificar la entrega de un producto seguro e inocuo al consumidor. Sin embargo, el consumidor y el cliente han evolucionado; el mundo moderno los ha despertado y han elevado sus niveles de exigencia. La gama de opciones que se tiene actualmente les permite exigir y escoger de acuerdo con criterios muy bien definidos de calidad en servicio, en producto y desarrollo sostenible, entre otros aspectos.

Esta necesidad de cumplir con los requisitos que demanda el mercado y el entorno ha generado entonces el surgimiento de múltiples modelos de mejoramiento y las organizaciones en todo el mundo han entrado en ese boom, llegando a implementar hasta cinco modelos de mejoramiento en las mismas [2].

Por lo anterior, las organizaciones buscan la integración de los sistemas de gestión, entendidos como el conjunto de elementos relacionados o que interactúan y permiten implantar y alcanzar la política y los objetivos de una organización, en cuanto a calidad, medio ambiente, seguridad y salud, u otras disciplinas de gestión [3].

Uno de estos nuevos modelos de gestión para las plantas manufactureras de alimentos es el FSSC22000-1, el cual deberá integrarse a los sistemas de gestión ya existentes. Este fue desarrollado en Europa y surgió del interés de grandes compañías como Nestlé, Danone, Unilever, Coca Cola, Kraft Products, entre muchas otras, de adoptar un nuevo modelo de certificación en inocuidad para todos sus proveedores de materias primas [4]; es decir, que implementar HACCP de forma directa o bajo la norma ISO 22000 ya no es suficiente, si el interés es competir con las grandes compañías. Este modelo de certificación de inocuidad de los alimentos está basado en la integración de la Norma ISO 22000 y la guía de pre-requisitos generales BSI-PAS 220. El estándar ha sido promovido por la Confederación Europea de Industrias de Alimentación y Bebidas (CIAA), y posteriormente aprobado por la GFSI (Iniciativa Global de Seguridad Alimentaria) [5].

La GFSI surge en el año 2000 de la necesidad de mejorar la seguridad de los alimentos, de garantizar la protección del consumidor y de la importancia de fortalecer su confianza, a través de fijar normas para los planes de seguridad alimentaria y potenciar la rentabilidad en todas las fases de la cadena del suministro de los alimentos. Dentro de sus objetivos está el mejorar la eficiencia de costos en toda la cadena de suministro 
alimentario mediante la aceptación común de las normas GFSI reconocidas por los minoristas en todo el mundo [6].

De acuerdo con la lista presentada por Food Safety System Certification 22000, fundación encargada de administrar el modelo en el mundo, las empresas que se certificaron en el primero año de vigencia de la norma (2009) fueron 12, y seis años después de desarrollado el modelo ya se cuenta con 10.595 organizaciones certificadas, distribuidas en 163 países [7].

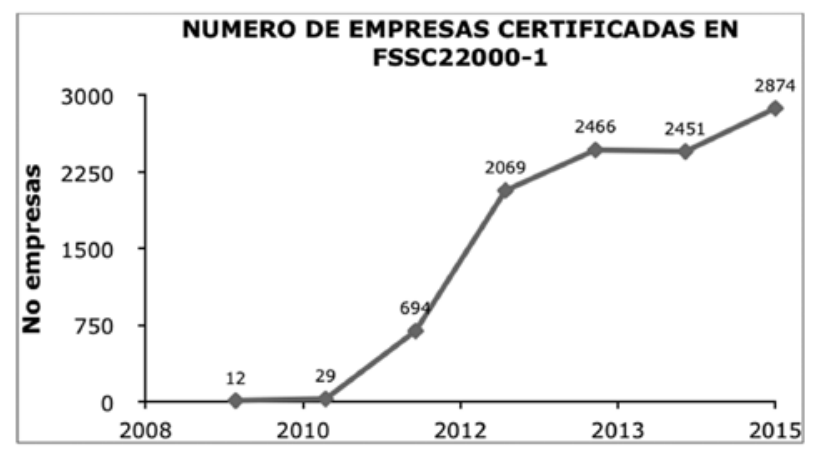

Fig. 1 Organizaciones certificadas año 2009 a septiembre de 2015.

Los datos anteriores ponen en evidencia la acogida que ha tenido FSSC22000-1 y el número creciente de organizaciones que lo han adoptado como estrategia para asegurar la inocuidad de los productos que entregan a sus clientes y consumidores.

Este sistema también genera interés para los países que quieren fortalecer los tratados de libre comercio entre naciones ya que sus organizaciones localmente estarían preparadas para competir y convertirse en ventaja competitiva. De acuerdo con la información suministrada por parte de la Food Safety System Certification 22000 , sobre la lista de empresas certificadas a la fecha de estudio, [7], se establece que la organización con mayor número de plantas certificadas es Nestlé (285); le siguen Cargill(170),
Mondelez (109), Unilever (104) y en quinto lugar Coca Cola (72).

Mundialmente, Estados Unidos lidera la lista de organizaciones certificadas y se ubica dentro de los diez primeros; además, Japón (857), China (820), India (496), México (472), Países Bajos (411), Alemania (321), Rusia (319), Sudáfrica (293) e Italia (208) engloban el $48 \%$ de las compañías que han adoptado el sistema FSSC22000-1. América Latina representa solo el $10,9 \%$ de acuerdo con el número de compañías certificadas a la fecha del estudio, siendo México el país con mayor número (472), seguido de Brasil (266), Argentina (131) Costa Rica (82) y Chile (41). A partir de allí, cada país aporta entre 37 y 4 compañías certificadas como máximo, a saber, Colombia, Perú, Ecuador y Guatemala, entre otros [7].

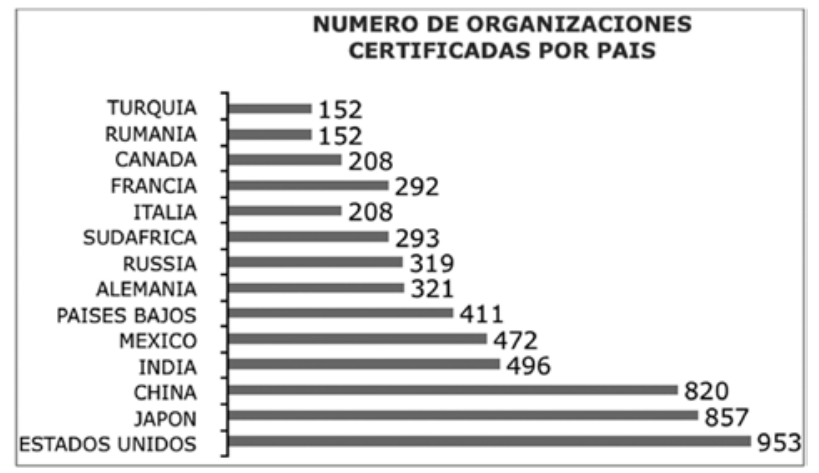

Fuente: FSSC, 2013. Organizaciones certificadas FSSC22000-1 Consultado 28 sept. 2015 
Colombia, a la fecha de la consulta (28 sept.2015), contaba con 38 plantas procesadoras certificadas: proveedores de material de empaque, de sabores, café soluble, grasas y aceites, envasadoras de gaseosas e ingenios azucareros, entre otras[7]. En el país se cuenta con varios organismos certificadores avalados por la fundación FSSC, entre ellos, Icontec y SGS [8]. Al entrevistar a la líder de la implementación del modelo FSSC22000 en una de las compañías certificadas, sobre las razones que los llevaron a tomar la decisión de acreditarse bajo este esquema, se pone de manifiesto la necesidad de contar con una certificación que tenga reconocimiento global y que brinde seguridad a los clientes a través de un estándar integral con herramientas para gestionar la inocuidad y además lograr la reducción asociada a las auditorías de segunda parte. Dentro de las mayores dificultades para alcanzar la certificación se menciona la baja disponibilidad de auditores con competencia y acreditación en todos los sectores manufactureros y los costos de inversión en obras civiles.

\section{Metodología}

Para la recopilación de los documentos bibliográficos se utilizaron tanto fuentes primarias como secundarias. Como fuentes primarias de información se usó la base de datos de organizaciones certificadas en el modelo de inocuidad, de la fundación FSSC, se entrevistó a la líder encargada de poner en funcionamiento el modelo en una de las compañías certificadas dedicada a la fabricación de café soluble para exportación y se tuvieron en cuenta las experiencias de la autora en la implementación de los programas prerrequisitos de buenas prácticas de manufactura (BPM) establecidas en el Decreto 3075 de 1997 y el Decreto 60 de 2002 sobre la implementación del Sistema de Análisis de Peligros y Puntos de Control Crítico HACCP.

En cuanto a la consecución de las fuentes secundarias se realizó una búsqueda entre el último trimestre del año 2011 y septiembre de 2015, seleccionando aquellos documentos que informasen sobre los conceptos y fundamentos teóricos del sistema objeto de este estudio, su evolución y la acogida que ha tenido en las diferentes organizaciones y países.

La metodología propuesta recoge entonces dos elementos fundamentales para su desarrollo: la experiencia de la autora en implementación de sistemas de gestión y la recopilación de fuentes documentales de diferentes autores y organizaciones.

\section{DesarRollo}

FSSC22000 ha sido elaborado para certificar los sistemas de inocuidad de las organizaciones que procesan o fabrican productos de origen animal, productos vegetales perecederos, productos con larga vida útil, ingredientes alimenticios como aditivos, vitaminas y cultivos biológicos, así como materiales para el empacado de alimentos [9]; incluye transporte y almacenamiento en el sitio, si este hace parte de la operación. Es aplicable a todas las plantas de manufactura, independiente del tamaño, complejidad y tipo de organización (pública o privada) [10].

Respecto del surgimiento de este sistema de gestión de la inocuidad alimentaria, la GFSI (Iniciativa Mundial de Seguridad Alimentaria) está patrocinada por CIES, una organización que engloba alrededor de 350 empresas con presencia en 150 países y representa alrededor del $75 \%$ de la distribución mundial y de sus proveedores [11].

En el momento en que la GFSI evaluó la ISO 22000 para su consideración y aceptación dentro de la lista de estándares establecidos, no identificó que estuvieran cubiertos en detalle los programas requisito necesarios para satisfacer de forma específica los requerimientos de inocuidad para el procesamiento de alimentos (plantas de producción), ya que la ISO 22000 en su sección 7.2.3 exige explícitamente la aplicación de programas de requisitos previos, y proporciona una lista de temas por considerar, pero no especifica qué debería incluir un Programa prerrequisito o PRP [9]. 
Esto llevó a elaborar el PAS 220, el cual fue diseñado para usarse en unión o como complemento de ISO 22000; de allí lo que se conoce como FSSC22000. La PAS 220 (o ISO/TS 22002-1) se desarrolló bajo el patrocinio de la BSI, a través de la CIAA y de 4 empresas multinacionales: Kraft, Danone, Unilever y Nestlé [12]La FSSC22000 está regulada por una fundación radicada en Suiza, conocida como FFSC (Foundation Food Safety Certification). Dentro del marco de las actualizaciones hechas por esta Fundación para regular las categorías que pueden certificarse en el esquema FSSC 22000, se ha ampliado el alcance de tal manera que ya se encuentra la autorización para empresas que están dentro de la categoría "M" (fabricación de material de empaque), y evidencien el cumplimiento de los requerimientos estipulados por PAS223:2011. De acuerdo con lo anterior, las organizaciones que certifiquen su sistema de inocuidad para la producción de alimentos lo harán con la norma FSSC22000-1 y las organizaciones relacionadas con la fabricación de materiales para empacado lo harán con la norma FSSC22000-4 [13].

\section{A. Estructura de FSSC22000}

Como se ha mencionado, FSSC22000 está basado mayoritariamente en ISO 22000 y PAS 220. Ver Fig. 3.

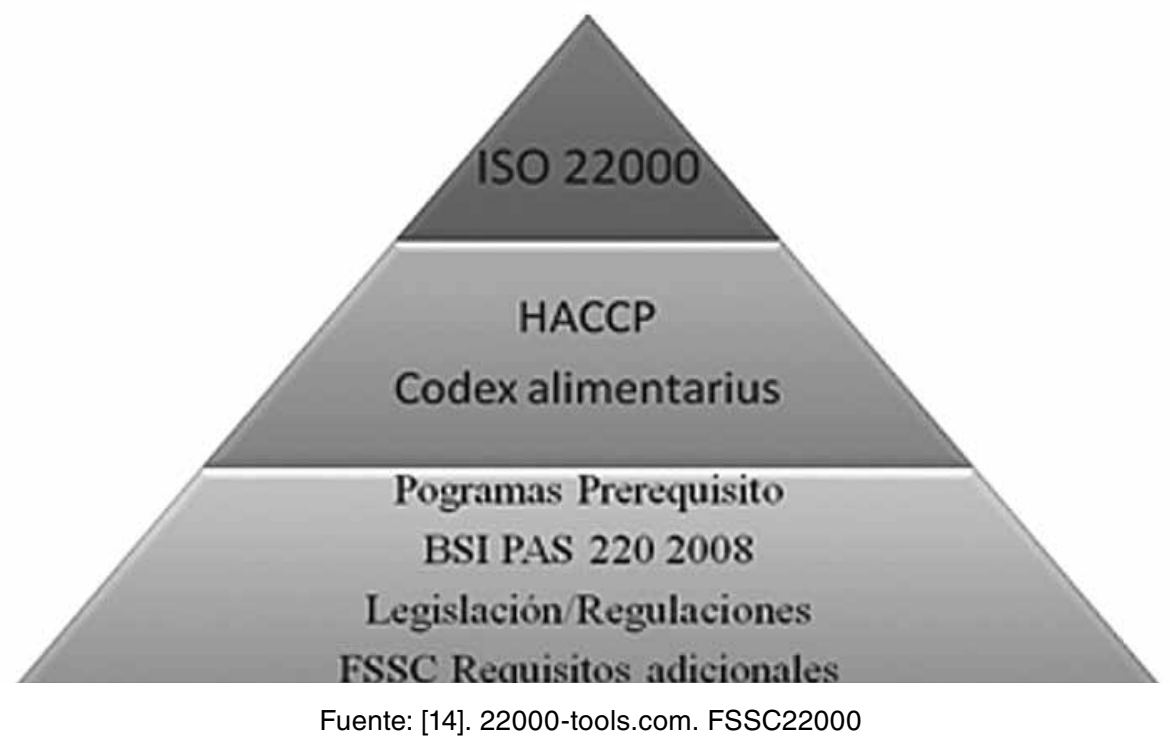

Fig. 3 Requisitos para implementación de FSSC22000.

A continuación se presentan los ítems que corresponden a cada uno de estos estándares:

1) Estructura de la ISO 22000:2010. El objetivo más importante para la ISO 22000 es identificar todas las condiciones básicas y necesarias para mantener bases permanentes de condiciones higiénicas durante la producción, manipulación, almacenamiento y suministro de alimentos inocuos para el consumo humano [15].
Esta norma está dividida en 8 secciones [16], de las cuales las secciones 1 a 3 corresponden a información general y las secciones 4 a 8 establecen los requisitos.

\section{Sección 1. Alcance}

Sección 2. Referencias normativas.

Sección 3. Términos y definiciones.

Sección 4. Sistema de gestión de seguridad alimentaria. Incluye requisitos generales (Sección 4.1) y requisitos de documentación (Sección 4.2). 
Sección 5. Responsabilidad de la dirección. Incluye la definición del compromiso de la dirección (5.1), política de inocuidad (5.2), planificación (5.3), responsabilidad y autoridad (5.4), líder del equipo de inocuidad (5.5), comunicación interna y externa (5.6), preparación y respuesta a emergencias (5.7), revisión por la dirección (5.8).

\section{Sección 6. Gestión de recursos.}

Sección 7. Planificación y realización de productos seguros. El alcance de los principios de esta sección va desde el diseño del producto hasta su realización.
La organización tendrá que planificar todos los procesos involucrados a fin de garantizar un producto final inocuo. Allí se incluye: el programa de pre-requisitos (7.2) el cual debe ser implementado usando como guía PAS 220 (o el que aplique según la industria específica) y ser aprobado por el equipo de inocuidad de la organización; etapas preliminares para realizar el análisis de peligros (7.3), análisis de peligros (7.4), establecer los programas pre-requisito operacionales (7.5), establecer el plan HACCP (7.6), actualización (7.7), planificación de la verificación (7.8), trazabilidad (7.9), control de la no conformidad (7.10).

Sección 8: validación, verificación y mejora del sistema de seguridad alimentaria.

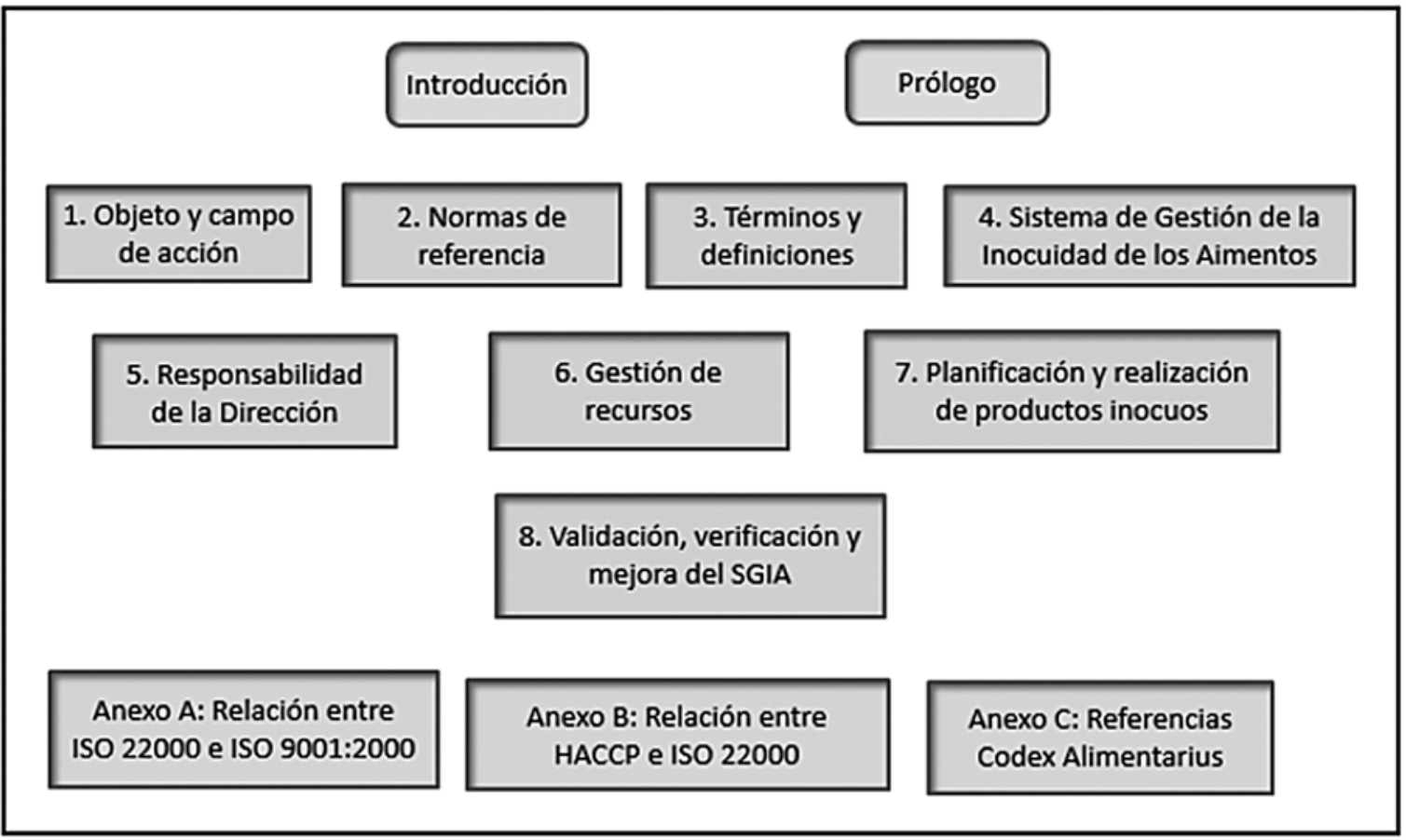

Fig. 4 Estructura de la norma ISO 22000.

2) Estructura de PAS 220:2008. EI PAS, disponible públicamente, especifica requerimientos para establecer, implementar y mantener programas prerrequisito (PPR) con el ánimo de ayudar en el control de peligros de los alimentos y su seguridad. Este es aplicable a todas las organizaciones (independiente de su tamaño o complejidad) que están involucradas en la etapa de producción de la cadena de alimentos y desean implementar PPR de tal manera de hacer frente a los requerimientos especificados en BS en ISO 22000:2205 cláusula 7 [18].

Este estándar, al igual que ISO 22000, incluye en las cláusulas o secciones 1 a 3 información general y en las cláusulas 4 a 8 , los requisitos [19]: 
Cláusula 1. Alcance

Cláusula 2. Referencias normativas.

Cláusula 3. Términos y definiciones.

Cláusula 4. Construcción y planos de edificios.

Cláusula 5. Planificación de edificios y espacios.

Cláusula 6. Servicios - aire, agua, energía

Cláusula 7. Drenajes

Cláusula 8. Idoneidad de los equipos, limpieza y mantenimiento.

Cláusula 9. Gestión de materiales.

Cláusula 10. Medidas para la prevención de la contaminación cruzada.

Cláusula 11. Limpieza y desinfección.

Cláusula 12. Control de plagas.

Cláusula 13. Higiene personal e instalaciones de empleados.

Cláusula 14. Retrabajo

Cláusula 15. Procedimiento de retirada de producto.

Cláusula 16. Almacenamiento

Cláusula 17. Información del producto/ sensibilización a los consumidores.

Cláusula 18. Prevención de sabotajes, biovigilancia y bioterrorismo.

\section{B. Proceso de certificación FSSC22000}

FSSC 22000 requiere que se cumpla con lo siguiente:

- Sistema de Gestión de la Inocuidad Alimentaria ISO 22000

- Programas de prerrequisitos PAS 223

- Requerimientos adicionales (3 requisitos adicionales)

El proceso de certificación consiste de 6 pasos:
Paso 1: se genera una propuesta desde la casa certificadora, basada en el tamaño y la naturaleza de la organización. Una vez que es aceptada, el proceso de auditoría puede comenzar.

Paso 2: Luego existe una etapa opcional de "pre-auditoría.

Paso 3: se ejecuta la primera parte de la auditoría formal o Fase 1 - Revisión de la preparación: allí se evalúa in situ el cumplimiento del sistema de documentación de la organización con los requisitos de la norma.

Paso 4: fase 2 del proceso de auditoría inicial. La auditoría incluye entrevistas con los trabajadores y examen de los registros. Las observaciones de las prácticas de trabajo determinan el grado de cumplimiento real de los procesos con la norma y con el sistema de documentación propio de la organización. Una vez que las no conformidades se han abordado, un gerente de certificación realiza una revisión técnica de la auditoría para confirmar la emisión de un certificado.

Paso 5: Las visitas de seguimiento se programarán en intervalos de seis o doce meses. Durante las estas, se revisa la implementación de los planes de acción, frente a las últimas no conformidades, y se examinan ciertas partes obligatorias y otras seleccionadas del sistema de acuerdo con el plan de auditoría.

Paso 6: poco antes del tercer año de la certificación inicial, se realiza una visita de seguimiento para permitir una auditoría de recertificación. Luego las visitas de seguimiento continúan como antes, en un ciclo de tres años [20]. 


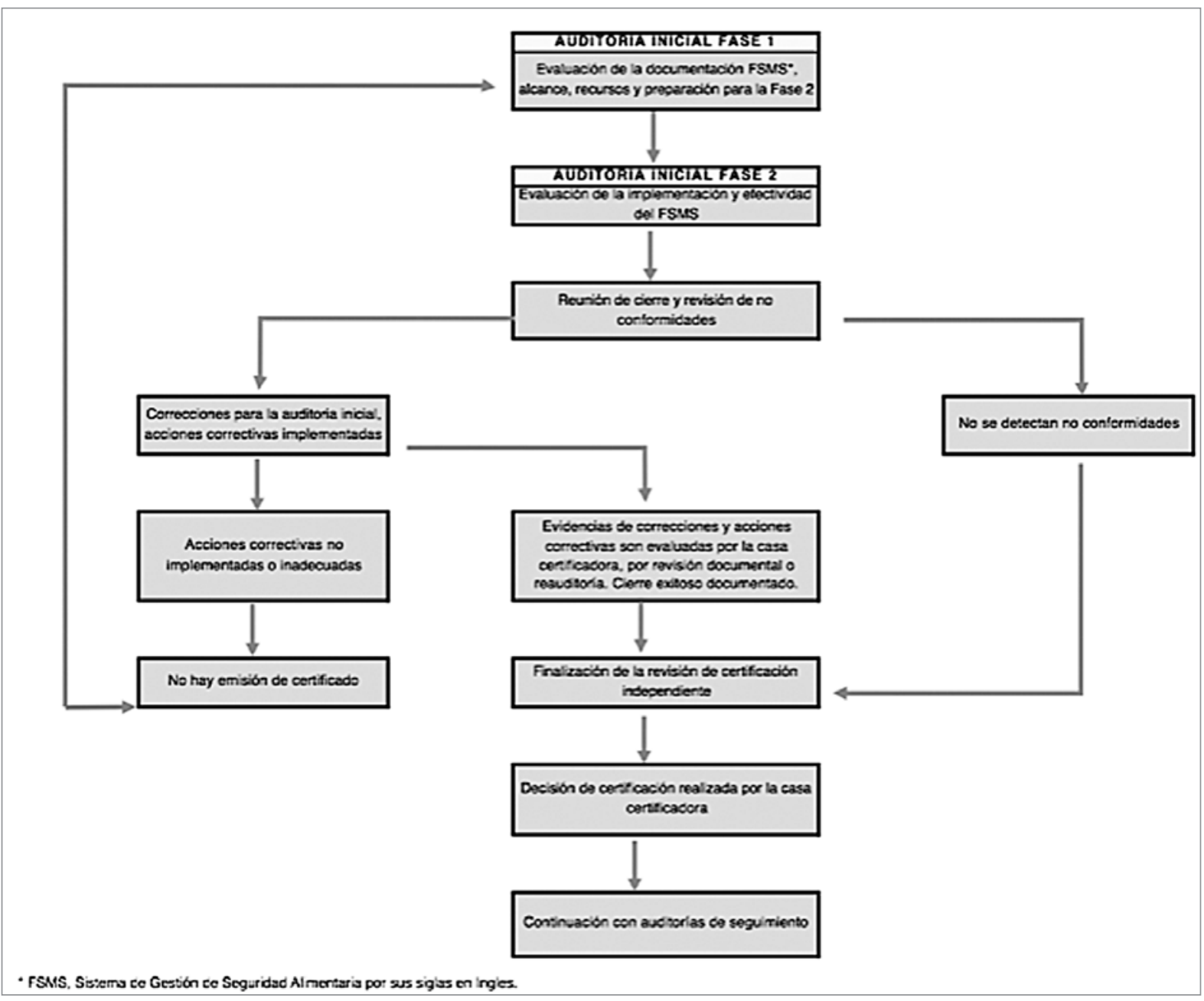

Fuente: Sansawat. 2011. Revisión de los estándares de gestión de inocuidad alimentariade diseño y fabricación de envases para alimentos

Fig. 5 Proceso de certificación FSSC 22000.

\section{CONCLusiones}

La implementación de FSSC22000 es de gran interés nacional e internacional tanto para las organizaciones que buscan ser proveedores de las grandes compañías manufactureras como Nestlé, Coca cola, Danone, entre muchas otras, como para los países que quieren fortalecer los tratados de libre comercio entre naciones ya que sus organizaciones localmente estarían preparadas para competir y convertirse en ventaja competitiva.

FSSC22000 ha sido elaborado para certificar los sistemas de inocuidad de las organizaciones que procesan o fabrican productos de origen animal, productos vegetales perecederos, productos con larga vida útil, ingredientes alimenticios como aditivos, vitaminas y cultivos biológicos, así como materiales para el empaque de alimentos. Vale aclarar que es aplicable a todas las plantas de manufactura, independientemente del tamaño, complejidad y tipo de organización.

De acuerdo con la lista presentada por Food Safety System Certification 22000, fundación encargada de administrar el modelo mundialmente desde el año 2009, fecha en que entró en vigencia el sistema, ya se cuenta con 10.595organizaciones certificadas, distribuidas en 163 países, lo cual pone en evidencia la acogida que ha tenido 
FSSC22000-1 y el número creciente de organizaciones que lo han adoptado como estrategia para asegurar la inocuidad de los productos que entregan a sus clientes y consumidores.

En la estadística de organizaciones certificadas en América Latina, Colombia ocupa el quinto lugar con 38 plantas de producción certificadas en diversas líneas para alimentos como material de empaque, sabores y aromas, café soluble, grasas y aceites, ingenios azucareros y envasadoras de gaseosas, principalmente.

\section{Referencias}

[1] Anandappa, M. 2013. Evaluating food safety systems development and implementation by quantifying HACCP training durability. Theses and Dissertations-Animal and Food Sciences. Paper 19. Disponible en: http://uknowledge.uky.edu/cgi/viewcontent.cgi?articl $\mathrm{e}=1018 \&$ context=animalsci_etds.

[2] Mensah, L. 2011. Implementation of food safety management systems in the UK. Food Control. Vol. 22 pág. 1216-1225.

[3] AEC, 2011. Asociación Española para la Calidad. Integración de sistemas de gestión. Consultado 7 ene de 2012. Disponible en http://www.aec.es/web/guest/centro-conocimiento/integracion-de-sistemas-de-gestion.

[4] SGS. (2011). Curso Sistema de certificación alimentaria FSCC22000 y PAS 220. Ibagué, Colombia.13 pág.

[5] Compeau, E. 2013. Supplier assesment: A commitment of food safety. Kansas State University. Consultado: Sept 25 de 2015. Disponible en: http://krex.kstate.edu/dspace/bitstream/handle/2097/16243/ ElizabethCompeau2013.pdf?sequence $=5$.

[6] Nava, J.2011. The Global Food Safety Inictiative, GFSI. Memorias de $2^{\circ}$ Food Safety; seminario taller en inocuidad y calidad de alimentos. Disponible en: http:// www.foodsafety.com.co/pdf/memorias/2011/13_Joaquin_Nava_Tarde_GRUPO_DELCEN.pdf.

[7] FSSC 22000 (Food Safety System Certification 22000, NL). 2015. Base de datos de Organizaciones certificadas. (en línea). Países Bajos, NL. Consultado 28 sept 2015. Disponible en https://viasyst.net/fssc.

[8] Valdes, L. 2013. ICONTEC. La seguridad alimenta- ria. Memorias evento Tecnicaña. Disponible en: http:// www.tecnicana.org/pdf/eventos/2013/memorias_inocuidad/icontec.pdf.

[9] [9]

[10] LRQA, US. Lloyd's Register Quality Assurance.ISO 22000; Food Safety. Consultado 13 Dic. 2011. Disponible en http://www.lrqa.com/standards-and-schemes/iso22000/.

[11] Diez, O. 2010. La certificación de sistemas de gestión en seguridad alimentaria. Eroski Consumer. Consultado 13 dic. 2011. Disponible en http://www. consumer.es/seguridad-alimentaria/ciencia-y-tecnologia/2005/11/22/21256.php.

[12] FSC 2000. 2010. Esquema de certificación para sistema de inocuidad de alimentos de conformidad con ISO 22000:2005 y BSI-PAS 220:2008 27 pág.

[13] FFSC 22000 (Foundation for Food Safety Certification, NL). 2013. Foundation for Food Safety Certification. (en línea). Países Bajos, NL. Acerca de la FSSC 22000. Consultado 16 dic. 2013. Disponible en http:// www.fssc22000.com/sp/page.phpl.

[14] Vinca, MN. 2011. 22000-tools.com. FSSC22000. (en línea). Consultado 9 ene 2012. Disponible en http:// www.22000-tools.com/what-is-fssc-22000.html.

[15] Boutou, O. 2008. Eight keys to successful ISO 22000 implementation. ISO Management Systems. Consultado: Sept. 15 de 2015. Disponible en: http://www.iso. org/iso/livelinkgetfile-isocs?nodeid=15020097.

[16] ISO, 2010. ISO 22000: Sistemas de gestión de seguridad alimentaria-Requisitos de cualquier organización en la cadena alimentaria. 35 pág.

[17] Pertz, G. 2011. El sistema FSSC22000. G.A. Pertz Consultores. Consultado 10 feb 2012. Disponible en: http://www.slideshare.net/pertzgonzalo/el-sistemafssc-22000.

[18] BSI, 2008. British Standards Institution. PAS 220:2008. Programas prerequisito en materia de seguridad alimentaria para la fabricación de alimentos. Consultado 28 sept 2015. Disponible en: https:// sceqa.files.wordpress.com/2014/05/pas-220-espac3b1ol.pdf.

[19] BSI, 2012. PAS 220 Food Safety. (en línea) Consultado 7 ene 2012. Disponible en: http://www.bsigroup. com/en/Assessment-and-certification-services/management-systems/Standards-and-Schemes/PAS-220/.

[20] Sansawat, S. 2011. Revisión de los estándares de gestión de inocuidad alimentaria y de diseño y fabricación de envases para alimentos. SGS. http:// www.sgs.co/ /media/Global/Documents/White\%20 Papers/sgs-packaging-food-safety-white-paper-a4es-11-v1.ashx. 
